\title{
THE DUAL DYNAMIC PROGRAMMING
}

\author{
ANDRZEJ NOWAKOWSKI
}

(Communicated by Barbara L. Keyfitz)

\begin{abstract}
The dual approach to dynamic programming for the generalized problem of Bolza is described. A suitable verification theorem is proved and a dual optimal feedback control is introduced.
\end{abstract}

\section{INTRODUCTION}

We consider the optimal control problem of Bolza

$$
\operatorname{minimize} J(x, u)=\int_{a}^{b} L(t, x(t), u(t)) d t+l(x(b))
$$

subject to

$$
\begin{gathered}
\dot{x}(t)=f(t, x(t), u(t)) \quad \text { a.e. in }[a, b], \\
u(t) \in U(t), \quad t \in[a, b], \\
x(a)=c .
\end{gathered}
$$

Here $f:[a, b] \times R^{n} \times R^{m} \rightarrow R^{n}, L:[a, b] \times R^{n} \times R^{m} \rightarrow R, l: R^{n} \rightarrow R \cup\{+\infty\}$ are given functions, $c$ is a point in $R^{n}, U:[a, b] \rightarrow R^{m}$ is a multi-function (i.e., $U(t)$ is a subset of $R^{m}$ for each $t$ in $\left.[a, b]\right), x:[a, b] \rightarrow R^{n}$ is an absolutely continuous function, and $u:[a, b] \rightarrow R^{m}$ is a Lebesgue measurable function.

In order that problem (1)-(4) make sense, throughout the paper we assume the following basis hypothesis:

For each $s$ in $R^{n}$, the functions $(t, u) \rightarrow L(t, s, u),(t, u) \rightarrow f(t, s, u)$ are $(\mathbb{L} \times \mathbb{B})$-measurable, i.e., measurable with respect to the $\sigma$-algebra of subsets of $[a, b] \times R^{m}$, generated by products of Lebesgue sets in $[a, b]$ and Borel sets in $R^{m}$. There exists functions $k_{1}, k_{2}$ in $L^{1}(a, b)$ such that for $t$ in $[a, b]$, $u$ in $U(t)$, and $s_{1}, s_{2}$ in $R^{n}$,

$$
\begin{aligned}
\left|L\left(t, s_{1}, u\right)-L\left(t, s_{2}, u\right)\right| & \leq k_{1}(t)\left|s_{1}-s_{2}\right|, \\
\left|f\left(t, s_{1}, u\right)-f\left(t, s_{2}, u\right)\right| & \leq k_{2}(t)\left|s_{1}-s_{2}\right| .
\end{aligned}
$$

The set $\left\{(t, u) \in[a, b] \times R^{m}: u \in U(t)\right\}$ is $(\mathbb{L} \times \mathbb{B})$-measurable. The function $l$ is lower semicontinuous and not identically $+\infty$. A pair $x(t), u(t)$

Received by the editors September 24, 1990 and, in revised form, April 25, 1991.

1991 Mathematics Subject Classification. Primary 49C20. 
is admissible if it satisfies (2), (3), $L(t, x(t), u(t))$ is summable, and $l(x(b))$ is finite; then the corresponding trajectory $x(t)$ will simply be called admissible.

For that problem, in [7] two methods of construction of fields of extremals (concourses of flights) were described and, as a consequence of this, sufficient conditions for optimality in a form similar to Weierstrass's were formulated. In remarks, there were given relations of these two theories to the dynamic programming technique. The first (classical) is the following: (see, e.g., [1, 3, $4,6]$ ) define the value function $S(t, x)$ in a set $T \subset R^{n+1}$ (being a set covered by graphs of trajectories of the field-concourse of flights) as

$$
S(t, x)=\inf \left\{\int_{t}^{b} L(t, x(\tau), u(\tau)) d \tau+l(x(b))\right\}
$$

where the infimum is taken over admissible pairs $x(\tau), u(\tau), \tau \in[t, b]$, whose trajectories start at $(t, x) \in T$ and graphs are contained in $T$. By [7, Theorem 4], the existence of value function (5) is determined by the existence of a concourse of flights (field of extremals). Further, it was concluded that if $S(t, x)$ is differentiable then it satisfies the partial differential equation

$$
S_{t}(t, x)+H\left(t, x, S_{x}(t, x)\right)=0,
$$

where $H(t, x, y)=y f(t, x, u(t, x))+L(t, x, u(t, x))$ and $u(t, x)$ is an optimal feedback control, and the partial differential equation of dynamic programming

$$
\min \left\{S_{t}(t, x)+S_{x}(t, x) f(t, x, u)+L(t, x, u): u \in U(t)\right\}=0 .
$$

In [7, Remark 4.2] the author suggested the second nonclassical approach to dynamic programming; the domain of exploration was carried out from the $(t, x)$-space to the space of multipliers $\left(\left(t, y^{0}, y\right)\right.$-space $)$. Then another function was defined-the dual value function- $S_{D}(t, p)$ in a set $P \subset R^{n+2}$ of the dual space $\left(t, y^{0}, y\right)=(t, p), y^{0} \leq 0$,

$$
S_{D}(t, p)=\inf \left\{-y^{0} \int_{t}^{b} L(\tau, x(\tau), u(\tau)) d \tau-y^{0} l(x(b))\right\}
$$

where the infimum is taken over admissible pairs $x(\tau), u(\tau), \tau \in[t, b]$, whose trajectories start at $(t, x(t, p))(x(t, p)$ will be defined below), and their graphs are contained in $T$ (defined above). By [7, Corollary $5^{\prime}$ ], the existence of $S_{D}(t, p)$ is determined by the existence of a concourse of flights. Next, a new function was defined: $V(t, p)=-S_{D}(t, p)-x(t, p) y=V_{y^{0}}(t, p) y^{0}+$ $V_{y}(t, p) y=V_{p}(t, p) p \quad\left(-S_{D}(t, p)=V_{y^{0}}(t, p) y^{0},-x(t, p)=V_{y}(t, p)\right),(t, p)$ $\in P$, which satisfies the partial differential equation

$$
V_{t}(t, p)+H\left(t,-V_{y}(t, p), p\right)=0,
$$

where $H(t, v, p)=y^{0} L(t, v, u(t, p))+y f(t, v, u(t, p))$ and $u(t, p)$ is a dual optimal feedback control, and the dual partial differential equation programming (briefly, DPDEDP)

$$
\max \left\{V_{t}(t, p)+y f\left(t,-V_{y}(t, p), u\right)+y^{0} L\left(t,-V_{y}(t, p), u\right): u \in U(t)\right\}=0 .
$$

The aim of this article is to get some properties of the value function $S(t, x)$ by studying (8), (9), (10) directly, which to a certain degree is the opposite 
approach to that in [7] and wholly in the spirit of the dynamic programming technique, though it is quite a new method in the study of the value function.

Note that when examining $(8)-(10)$ instead of $(5)-(7)$, we need not require that the set $T$ has nonempty interior, or that $S(t, x)$ is differentiable in $T$. Moreover, we do not require that $y^{0}=-1$ or that the problem is calm, which is essential in the classical setting $(5)-(7)[2,5]$. This is very important for problems with constraints (see, e.g., [5]). Further, if problem (1)-(4) can be solved using (6), (7), i.e., e.g., [6, Theorem 7.1, Chapter 4], then it can be solved also by dual dynamic programming, i.e., Theorem 3.1 or Theorem 3.2. Indeed, $T$ then has nonempty interior and $S(t, x)$ is of class $C^{1}$ in an open subset $Q$ of $T$. Put $P=\left\{(t, p): a \leq t \leq b, p=\left(y^{0}, y\right),-2<y^{0}<0, y=y^{0} S_{x}(t, x)\right.$, $(t, x) \in Q\}$ and define $x(t, p),(t, p) \in P$ as the value of an optimal trajectory starting at $(t, x)$ for $(t, p)=\left(t, y^{0}, y^{0} S_{x}(t, x)\right)$. Then $-y^{0} V_{y^{0}}(t, p)=$ $S_{D}(t, p)=-y^{0} S(t, x(t, p)), V_{t}(t, p)=y^{0} S_{t}(t, x(t, p)),-V_{y}(t, p)=x(t, p)$. Putting these values in (7) multiplied by $y^{0}$, we find that $V(t, p)=V_{p}(t, p) p$ satisfies (10). Conversely if there exists a $V(t, p)=V_{p}(t, p) p$ and satisfying (10) and such that closure of $T=\left\{(t, x): x=-V_{y}(t, p), t \in[a, b]\right.$, $(t, p) \in P\}$ has nonempty interior in which $S(t, x)$ is of class $C^{1}$ and $y^{0}<0$, then from (10) we analogously get (7).

\section{The dual Value function AND The NeW function $V(t, p)$}

Let $T \subset R^{n+1}$ denote a set covered by the graphs of all admissible trajectories. We shall assume in this section, taking if necessary a smaller set $T$, that $S(t, x)$ defined in $T$ by (5) does not take the value $\pm \infty$. Now $T$ is, in general, larger than that defined in $\S 1$. Let $P \subset R^{n+2}$ be a set of variables $\left(t, y^{0}, y\right)=(t, p), t \in[a, b]$, with $y^{0} \leq 0$ and a nonempty interior. Take a function $x(t, p)$ defined on $P$ such that $(t, x(t, p)) \in T,(t, p) \in P$; we assume that it is measurable, locally bounded, and that for each admissible trajectory $x(t)$ lying in $T$ there exists a function of bounded variation $p(t)=\left(y^{0}, y(t)\right)$ lying in $P$ such that $x(t)=x(t, p(t))$ and if all trajectories $x(t)$ start at the same $\left(t_{0}, x_{0}\right)$, then all the corresponding $p(t)$ have the same first coordinate $y^{0}$.

Further, let $S_{D}(t, p)$ be as in (8) but with $T$ and $x(t, p)$ defined here. We see that $S_{D}(t, p)=-y^{0} S(t, x(t, p)),(t, p) \in P$. Following [6, Chapter IV.3] (compare [4, Chapter 5.4]), we get a modification of the known proposition.

Proposition 2.1. (i) For each function of bounded variation $p(t)=\left(y^{0}, y(t)\right)$, $t \in\left[t_{1}, b\right], a \leq t_{1}<b$, lying in $P$ such that $x(t)=x(t, p(t)), t \in\left[t_{1}, b\right]$, is an admissible trajectory lying in $T$, the function $\bar{W}(t, p(t))=S_{D}(t, p(t))+$ $y^{0} \int_{t}^{b} L(s, x(s), u(s)) d s$ is nondecreasing in $\left[t_{1}, b\right]$. If $\bar{p}(t)=\left(\bar{y}^{0}, \bar{y}(t)\right), t \in$ $\left[t_{1}, b\right]$, is absolutely continuous and is such that $\bar{x}(t)=x(t, \bar{p}(t)), t \in\left[t_{1}, b\right]$, is an optimal trajectory on $\left[t_{1}, b\right]$ with $x\left(t_{1}\right)=x\left(t_{1}, \bar{p}\left(t_{1}\right)\right)$, then $\bar{W}(t, \bar{p}(t))$ is constant in $\left[t_{1}, b\right]$.

(ii) Let $W(t, p)=-y^{0} Z(t, x(t, p))$ be a real-valued function in $P$ such that $W(b, p)=-y^{0} l(x(b, p))$. Let $\left(t_{0}, x_{0}\right) \in T$ be a given initial condition. Suppose that for each function of bounded variation $p(t)=\left(y^{0}, y(t)\right), t \in\left[t_{0}, b\right]$, with graph lying in $P$, the mapping $t \rightarrow x(t)=x(t, p(t)), t \in\left[t_{0}, b\right]$, $x\left(t_{0}\right)=x_{0}$, is an admissible trajectory lying in $T$ and that $W(t, p(t))+$ 
$y^{0} \int_{t}^{b} L(s, x(s), u(s)) d s(u(t)$ is feasible for $x(t))$ is nondecreasing on $\left[t_{0}, b\right]$. If $\bar{p}(t)=\left(\bar{y}^{0}, \bar{y}(t)\right), t \in\left[t_{0}, b\right]$, is absolutely continuous and if $\bar{x}(t)=x(t, \bar{p}(t))$, $t \in\left[t_{0}, b\right], \bar{x}\left(t_{0}\right)=x_{0}$, is an admissible trajectory in $T$, and is such that $W(t, \bar{p}(t))+y^{0} \int_{t}^{b} L(s, \bar{x}(s), \bar{u}(s)) d s$ is constant in $\left[t_{0}, b\right]$, then $\bar{x}(t)$ is an optimal trajectory and $W\left(t_{0}, \bar{p}\left(t_{0}\right)\right)=S_{D}\left(t_{0}, \bar{p}\left(t_{0}\right)\right)$ where $\bar{u}(t)$ is a control corresponding to $\bar{x}(t)$.

Proof. We only show part (ii). For any function $p(t), t \in\left[t_{0}, b\right]$, described above, $-y^{0} Z\left(t_{0}, x_{0}\right) \leq-y^{0} \int_{t_{0}}^{b} L(s, x(s), u(s)) d s-y^{0} l(x(b))$ where $u(t)$ is a control feasible for $x(t)$. For the function $\bar{p}(t)$,

$$
-\bar{y}^{0} Z\left(t_{0}, x_{0}\right)=-\bar{y}^{0} \int_{t_{0}}^{b} L(s, \bar{x}(s), \bar{u}(s)) d s-\bar{y}^{0} l(\bar{x}(b)),
$$

so $W\left(t_{0}, \bar{p}\left(t_{0}\right)\right)=S_{D}\left(t_{0}, \bar{p}\left(t_{0}\right)\right)$ and $\bar{x}(t), \bar{u}(t)$ is an optimal pair for the problem

$$
\min \left\{-\bar{y}^{0} \int_{t_{0}}^{b} L(s, x(s), u(s)) d s-\bar{y}^{0} l(x(b)): x(t), u(t), t \in\left[t_{0}, b\right],\right.
$$

$$
\text { admissible pairs with } \left.x\left(t_{0}\right)=x_{0} \text { and } x(t) \text { lying in } T\right\} \text {. }
$$

Now, assume the triplet $P, x(t, p), T$ can be chosen in such a way that if we put $V_{y^{0}}(t, p)=S(t, x(t, p)), V_{y}(t, p)=-x(t, p)$, then there exists a function $V(t, p)$ in $P$ satisfying

$$
\begin{aligned}
V(t, p) & =V_{p}(t, p) p=V_{y^{0}}(t, p) y^{0}+V_{y}(t, p) y \\
& =y^{0} S(t, x(t, p))-y x(t, p)
\end{aligned}
$$

with the convention: if $y^{0}=0$ then $V(t, p)=V_{y}(t, p) y$. In [7] it was shown that such a function can exist (see $\S 1)$.

Proposition 2.2. Assume in addition to $(\mathrm{H})$ that $U=U(t), t \in[a, b]$, is $a$ fixed subset of $R^{m}$ and $L, f$ are continuous in $[a, b] \times R^{n} \times R^{m}$. Moreover, suppose that if an admissible trajectory $x(t)$ is of class $C^{1}$ in a subinterval of $[a, b]$ then the corresponding $p(t) \quad(x(t)=x(t, p(t)))$ is also of class $C^{1}$ on this subinterval. Let $\left(t_{0}, p_{0}\right)=\left(t_{0}, y_{0}^{0}, y_{0}\right)$ be an interior point of $P$ at which the function $V(t, p)$ is differentiable and such that at $\left(t_{0}, x\left(t_{0}, p_{0}\right)\right)$ admissible trajectories under constant controls lying in $T$ may start. Then $V(t, p)$ satisfies at $\left(t_{0}, p_{0}\right)$ the dual partial differential inequality

$$
V_{t}\left(t_{0}, p_{0}\right)+y_{0} f\left(t_{0},-V_{y}\left(t_{0}, p_{0}\right), v\right)+y_{0}^{0} L\left(t_{0},-V_{y}\left(t_{0}, p_{0}\right), v\right) \leq 0
$$

for all $v \in U$.

If there is an optimal pair $\bar{x}(t), \bar{u}(t), t \in\left[t_{0}, b\right]$, for the problem $S\left(t_{0}, x\left(t_{0}, p_{0}\right)\right)$ (see $\left.(5)\right)$, with $\bar{u}(t)$ being continuous on some $\left[t_{0}, t_{0}+k\right]$, then the DPDEDP

$$
\max _{v \in U}\left\{V_{t}\left(t_{0}, p_{0}\right)+y_{0} f\left(t_{0},-V_{y}\left(t_{0}, p_{0}\right), v\right)+y_{0}^{0} L\left(t_{0},-V_{y}\left(t_{0}, p_{0}\right), v\right)\right\}=0
$$

is satisfied. 
Proof. Let $v \in U$ be any constant control used over an interval $\left[t_{0}, t_{0}+k\right]$ with $k$ small enough. Let $x(t), t \in\left[t_{0}, t_{0}+k\right]$, be a trajectory corresponding to $v$, starting at $x\left(t_{0}, p_{0}\right)$ and lying in $T$. Let $p(t)=\left(y^{0}, y(t)\right), t \in\left[t_{0}, t_{0}+k\right]$, be a suitable function for $x(t)$, lying in $P$. Denote by $\tilde{x}(t), t \in\left[t_{0}+k, b\right]$, an admissible trajectory starting at $x\left(t_{0}+k\right)$ and lying in $T ; \tilde{u}(t), t \in\left[t_{0}+k, b\right]$, is its control. Put

$$
\begin{aligned}
& x_{k}(t)= \begin{cases}x(t), & t_{0} \leq t \leq t_{0}+k, \\
\tilde{x}(t), & t_{0}+k \leq t \leq b,\end{cases} \\
& u_{k}(t)= \begin{cases}v, & t_{0} \leq t \leq t_{0}+k, \\
\tilde{u}(t), & t_{0}+k \leq t \leq b,\end{cases}
\end{aligned}
$$

and let $p_{k}(t)=\left(y^{0}, y_{k}(t)\right), t \in\left[t_{0}, b\right]$, be the corresponding function, i.e., $x_{k}(t)=x\left(t, p_{k}(t)\right), t \in\left[t_{0}, b\right]$. By Proposition 2.1(i),

$$
t \rightarrow \bar{W}(t, p(t))=-y_{k}^{0} S\left(t, x\left(t, p_{k}(t)\right)\right)+y_{k}^{0} \int_{t}^{b} L\left(\tau, x_{k}(\tau), u_{k}(\tau)\right) d \tau
$$

is nondecreasing, and so $(d / d t) \bar{W}(t, p(t)) \geq 0$ for any value of $t$ for which this derivative exists. Since $(d / d t) x_{k}(t)$ exists at $t=t_{0}$, by the assumption on $p(t), t \in\left[t_{0}, t_{0}+k\right]$, and (11), there exists $(d / d t) S\left(t, x\left(t, p_{k}(t)\right)\right)$ at $t=t_{0}$ and thus also $(d / d t) \bar{W}(t, p(t))$ at $t=t_{0}$. Hence $(d / d t) \bar{W}\left(t_{0}, p\left(t_{0}\right)\right) \geq 0$. Calculating the last derivative, using (11) and the relation $(d / d t) x\left(t_{0}, p_{k}\left(t_{0}\right)\right)=$ $f\left(t_{0}, x\left(t_{0}, p_{k}\left(t_{0}\right)\right), v\right)$, we get (12).

If $\bar{x}(t), \bar{u}(t), t \in\left[t_{0}, b\right]$, is an admissible optimal pair for $S\left(t_{0}, x\left(t_{0}, p_{0}\right)\right)$, then for $\bar{p}(t)$ corresponding to $\bar{x}(t)=x(t, \bar{p}(t)), t \in\left[t_{0}, b\right], \bar{W}(t, \bar{p}(t))$ is constant in $\left[t_{0}, b\right]$; thus $(d / d t) \bar{W}(t, \bar{p}(t))=0$ at $t=t_{0}$, and so $V_{t}\left(t_{0}, p_{0}\right)+$ $y_{0} f\left(t_{0},-V_{y}\left(t_{0}, p_{0}\right), \bar{u}\left(t_{0}\right)\right)+y_{0}^{0} L\left(t_{0},-V_{y}\left(t_{0}, p_{0}\right), \bar{u}\left(t_{0}\right)\right)=0$. This and (12) yield (13).

Remark 2.1. To prove this proposition, we do not require the differentiability of $S(t, x)$.

\section{A VERIFICATION THEOREM AND A DUAL OPTIMAL FEEDBACK CONTROL}

In this section we give an answer to the question: "When does a solution $V(t, p)$ of the DPDEDP satisfy the sufficient conditions for optimality in Proposition 2.1(ii)?"

Theorem 3.1. Let $V(t, p),(t, p) \in P, t \in[a, b]$, be a Lipschitz solution of $D P D E D P(10)$. Let $E$ denote a subset of $[a, b]$ such that if $t_{0} \in E$, then for all $\left(t_{0}, p\right) \in P, V_{p}(t, p)$ exists. We assume that meas $E=b-a, b \in E$, and that $V(t, p)$ satisfies the boundary condition $y^{0} V_{y^{0}}(b, p)=y^{0} l\left(-V_{y}(b, p)\right)$, $(b, p) \in P$, and the relation

$$
V(t, p)=V_{p}(t, p) p, \quad t \in E,(t, p) \in P .
$$

Let $x(t), u(t)$ be an admissible pair whose graph of the trajectory $x(t)$ is contained in the closure $\bar{T}$ of $T=\left\{(t, x): x=-V_{y}(t, p), t \in E,(t, p) \in P\right\}$ and such that there is a function of bounded variation $p(t)=\left(y^{0}, y(t)\right)$ lying in $P$ and satisfying $x(t)=-V_{y}(t, p(t))$ for $t \in E$. Assume further that 
then $V_{t}(t, p(t))$ exists for almost every $t$. Then $\bar{W}(t, p(t))=-y^{0} V_{y^{0}}(t, p(t))+$ $y^{0} \int_{t}^{b} L(s, x(s), u(s)) d s$ is a nondecreasing function of $t$. Let $\bar{x}(t), \bar{u}(t), t \in$ $[a, b], \bar{x}(a)=c$, be an admissible pair with $\bar{x}(t)$ lying in $\bar{T}$ and let $\bar{p}(t)=$ $\left(\bar{y}^{0}, \bar{y}(t)\right), t \in[a, b]$, be a nonzero absolutely continuous function lying in $P$ such that $\bar{x}(t)=-V_{y}(t, \bar{p}(t))$ for all $t \in E$. Suppose that for almost all $t$ in $[a, b]$,

(15) $V_{t}(t, \bar{p}(t))+\bar{y}(t) f\left(t,-V_{y}(t, \bar{p}(t)), \bar{u}(t)\right)+\bar{y}^{0} L\left(t,-V_{y}(t, \bar{p}(t)), \bar{u}(t)\right)=0$.

Then $\bar{x}(t), \bar{u}(t), t \in[a, b]$, is an optimal pair for (1)-(4) relative to all admissible pairs $x(t), u(t), t \in[a, b], x(a)=c$, whose graphs of trajectories are contained in $\bar{T}$ and where the corresponding function $p(t)=\left(\bar{y}^{0}, y(t)\right) \quad(x(t)=$ $\left.-V_{y}(t, p(t)), t \in E\right)$ is of bounded variation. Moreover, $-\bar{y}^{0} S(t, x(t, \bar{p}(t)))=$ $-\bar{y}^{0} V_{y^{0}}(t, \bar{p}(t))$ with $x(t, p)=-V_{y}(t, p)$ is the dual value function along $\bar{p}(t)$. Proof. By (14), $V_{t}(t, p(t))=y^{0}(d / d t) V_{y^{0}}(t, p(t))+y(t)(d / d t) V_{y}(t, p(t))$ a.e. Since $(d / d t) V_{y}(t, p(t))=-f\left(t,-V_{y}(t, p(t)), u(t)\right)$ and $(d / d t) y^{0} V_{y^{0}}(t, p(t))=$ $-(d / d t) \bar{W}(t, p(t))-y^{0} L\left(t,-V_{y}(t, p(t)), u(t)\right)$ a.e., from (10) we get that $(d / d t) \bar{W}(t, p(t)) \geq 0$ a.e. The above relations, written for $\bar{p}(t)$, together with equation (15), imply that $-\bar{y}^{0} V_{y^{0}}(t, \bar{p}(t))=-\bar{y}^{0} \int_{t}^{b} L(s, \bar{x}(s), \bar{u}(s)) d s-$ $\bar{y}^{0} l\left(-V_{y}(b, \bar{p}(b))\right)$ for $t \in E$; thus we can extend $-\bar{y}^{0} V_{y^{0}}(t, \bar{p}(t))$ to all $t \in$ $[a, b] \backslash E$ by assuming it equal to the right-hand side of the last equality. Hence we get that $\bar{W}(t, \bar{p}(t))=-\bar{y}^{0} l(\bar{x}(b))$ is constant in $[a, b]$. This, together with Proposition 2.1(ii), implies the assertion of the theorem.

Remarks to Theorem 3.1. (i) If we also assume that $V_{y y} \neq 0$ exists and is continuous, then for each admissible trajectory $x(t)$ whose graph is contained in $T$, we get that the corresponding $p(t)=\left(y^{0}, y(t)\right)$ is really of bounded variation.

(ii) If $V$ is a Lipschitz solution of (10) then it will be of form (14) when $V_{p p}(t, p) p=0,(t, p) \in P$.

(iii) Put $x(t, p)=-V_{y}(t, p), S(t, x(t, p))=V_{y^{0}}(t, p)$. Then (14) means that $\left(y^{0},-y\right)$ is a normal to the epigraph of $S(t, x)$ defined in $T$ at the point $(x(t, p), S(t, x(t, p)))$. This generalizes the classical results (if $S(t, x)$ is smooth and $\left.y^{0}=-1\right)$ that $-y=S_{x}$ (see, e.g., $[4,6]$ ).

(iv) We would like to stress that the $x(t, p)$ of $\S 2$ which appeared in an artificial way, in practice, is calculated from (10) and (14) by putting $x(t, p)=$ $-V_{y}(t, p)$.

In the classical dynamic programming, another problem, whose solution is even more useful in applications, is that of finding the optimal feedback or optimal control synthesis $u(t, x)$ (see $\S 1$ ). In $\S 1$ the concept of a dual feedback control was mentioned, too. Now, we describe this concept more carefully.

Let a Borel measurable function $u=u(t, p)$ from a set $R \subset R^{n+2}$ of the points $(t, p)=\left(t, y^{0}, y\right), t \in[a, b], y^{0} \leq 0$, into $U(t)$ be given. Then the differential equation

$$
\dot{x}=f(t, x, u(t, p))
$$

has many solutions $x(t, p)$ in $P$. We say that $u=u(t, p)$ is a dual feedback control if we can choose any solution $x(t, p)$ of $(16)$ such that for each admissible trajectory $x(t)$ lying in $T=\{(t, x): x=x(t, p),(t, p) \in P\}$, there 
exists a function of bounded variation $p(t)=\left(y^{0}, y(t)\right)$ lying in $P$ and satisfying $x(t)=x(t, p(t))$. A dual feedback $u(t, p)$ will be called optimal if for each $(t, p) \in P$ there exists an absolutely continuous function $\bar{p}(s)=\left(\bar{y}^{0}, \bar{y}(s)\right)$, $s \in[t, b]$, such that $S_{D}(t, p)=-y^{0} \int_{t}^{b} L(s, x(s, \bar{p}(s)), u(s, \bar{p}(s))) d s-$ $y^{0} l(x(b, \bar{p}(b)))$ and for $y^{0} V_{y^{0}}(t, p)=-S_{D}(t, p), V_{y}(t, p)=-x(t, p)$, there exists $V(t, p)$ satisfying (14) with a suitable set $E$. To obtain an optimal pair with an initial condition $\left(t_{0}, x_{0}\right) \in T$, we have to find an absolutely continuous function $\bar{p}(t)=\left(\bar{y}^{0}, \bar{y}(t)\right)$ lying in $P$ such that $x\left(t_{0}, \bar{p}\left(t_{0}\right)\right)=x_{0}$, $(d / d t) x(t, \bar{p}(t))=f(t, x(t, \bar{p}(t)), u(t, \bar{p}(t)))$ and along it (14) is satisfied with the above $V_{p}$ and the set $E$. The pair defined by $\bar{x}(t)=x(t, \bar{p}(t))$, $\bar{u}(t)=u(t, \bar{p}(t)), t \in\left[t_{0}, b\right]$, will be an optimal pair.

Theorem 3.2. Let $u(t, p)$ be a dual feedback control in $P$. Then a sufficient condition that $u(t, p)$ be optimal is that the following hold: the function $\bar{V}(t, p)=$ $y^{0} \int_{t}^{b} L(s, x(s, \bar{p}(s)), u(s, \bar{p}(s))) d s-x(t, p) y+y^{0} l(x(b, \bar{p}(b)))$, for a set $E \subset$ $[a, b]$ of full measure containing $t=b$, has the derivative $\bar{V}_{p}(t, p)$ for $t \in$ $E$ and $(t, p) \in P$ with $y^{0} \bar{V}_{y^{0}}(t, p)=y^{0} \int_{t}^{b} L(s, x(s, \bar{p}(s)), u(s, \bar{p}(s))) d s+$ $y^{0} l(x(b, \bar{p}(b))), \bar{V}_{y}(t, p)=-x(t, p), t \in E,(t, p) \in P$, and is a Lipschitz solution of DPDEDP (10) in $P . \bar{p}(s), s \in[t, b]$, is absolutely continuous and is chosen, for each $(t, p) \in P$, in such a way that $\bar{x}(s)=x(s, \bar{p}(s)), \bar{u}(s)=$ $u(s, \bar{p}(s)), s \in[t, b]$, is an admissible pair and (15) is fulfilled in $[t, b]$ a.e. with $V_{t}(s, \bar{p}(s))=\bar{V}_{t}(s, \bar{p}(s)), V_{y}(s, \bar{p}(s))=\bar{V}_{y}(s, \bar{p}(s))$. If $p(t)=\left(y^{0}, y(t)\right)$ is a function of bounded variation lying in $P$ and corresponding to some admissible trajectory $x(t)$ lying in $T$, then $\bar{V}_{t}(t, p(t))$ exists for almost every $t$.

Proof. The proof is a direct consequence of the verification Theorem 3.1.

To demonstrate the value of the presented theory, we give an example that is solved in [7] by the nonclassical field theory method and could not be treated by any other known method. We show below that this example can also be solved by using Theorem 3.2.

Example. Take in (1)-(4) $a=-1, b=\pi, L(t, x(t), u(t))=a(t) x^{2}(t)+$ $b(t) u^{2}(t), l(x(\pi))=0$ if $x(\pi)=0$ and $+\infty$ otherwise, $f(t, x(t), u(t))=$ $B(t) u(t), U(t)=[-1,1], t \in[-1, \pi], x(-1)=0$, where

$$
\begin{gathered}
a(t)=\left\{\begin{array}{ll}
-\frac{1}{2}, & 0 \leq t \leq \pi, \\
0, & -1 \leq t<0 ;
\end{array} \quad b(t)= \begin{cases}\frac{1}{2}, & 0 \leq t \leq \pi, \\
1, & -1 \leq t<0 ;\end{cases} \right. \\
B(t)= \begin{cases}1, & 0<t \leq \pi, \\
-1, & t \in I_{k 1} \cup I_{k 3}, \\
0, & t \in I_{k 2} \cup\{-1\} ;\end{cases} \\
I_{k j}=\left(-1+\left(\frac{1}{2}\right)^{3 k+j},-1+\left(\frac{1}{2}\right)^{3 k+j-1}\right], \\
j=1,2,3, k=0,1, \ldots,
\end{gathered}
$$

To find an optimal dual feedback control, we help ourselves by resolving the maximum principle (the necessary optimality conditions) for problem (1)-(4) 
(see [7, pp. 749, 750]). We calculate from it the following triplets $x(t), u(t)$, $p(t)=\left(y^{0}, y(t)\right)$ :

$$
\begin{gathered}
y^{0}=-e, \quad x\left(t, c_{1}\right)=c_{1} \sin t, \quad y\left(t, e c_{1}\right)=e c_{1} \cos t, \quad u\left(t, c_{1}\right)=c_{1} \cos t, \\
t \in[0, \pi], \quad c_{1} \in(-1,1), e \in\left(\frac{1}{2}, \frac{3}{2}\right) ; \\
y^{0}=-e, \quad x(t, e)=0, \quad y(t, e)=0, \quad u(t, e)=0, \quad t \in[-1, \pi] ; \\
y^{0}=-e, \quad x\left(t, c_{4}\right)=-\frac{c_{4}}{2} \int_{t}^{0} B^{2}(s) d s, \quad y\left(t, e c_{4}\right)=e c_{4}, \\
u\left(t, c_{4}\right)=\frac{c_{4}}{2} B(t), \quad t \in[-1,0], c_{4} \in(-1,1) .
\end{gathered}
$$

Define

$$
u\left(t, y^{0}, y\right)= \begin{cases}-\left(y / 2 y^{0}\right) B(t), & t \in[-1,0], y^{0} \in\left(-\frac{3}{2},-\frac{1}{2}\right), \\ 0, & y \in\left(-\frac{3}{2}, \frac{3}{2}\right), \\ -\left(y / y^{0}\right), & t \in[-1, \pi], y^{0} \in\left(-\frac{3}{2},-\frac{1}{2}\right), y=0, \\ & t \in[0, \pi], y^{0} \in\left(-\frac{3}{2},-\frac{1}{2}\right), \\ & |y|<\frac{3}{2}|\cos t|, y=0 \text { for } t=\pi / 2 .\end{cases}
$$

Next, define $x\left(t, y^{0}, y\right)$ and $\bar{V}\left(t, y^{0}, y\right)$ in the same sets of $t$ and $\left(y^{0}, y\right)$, respectively, as

$$
\begin{gathered}
x\left(t, y^{0}, y\right)=\left\{\begin{array}{l}
\left(y / 2 y^{0}\right) \int_{t}^{0} B^{2}(s) d s, \\
0, \\
\left(-y / y^{0}\right) \operatorname{tg} t
\end{array}\right. \\
\bar{V}\left(t, y^{0}, y\right)=\left\{\begin{array}{l}
\left(-y^{2} / 4 y^{0}\right) \int_{t}^{0} B^{2}(s) d s, \\
0, \\
\left(y^{2} / 2 y^{0}\right) \operatorname{tg} t .
\end{array}\right.
\end{gathered}
$$

The set $E=[-1, \pi] \backslash\{\pi / 2\}$. It is not difficult to check that all assumptions of Theorem 3.2 are satisfied for the above $u(t, p), x(t, p), \bar{V}(t, p)$, and $E$. Thus $u(t, p)$ defined by (17) is an optimal dual feedback control and $x(t)=0$, $u(t)=0$ is an optimal pair.

\section{REFERENCES}

1. R. Bellman, Dynamic programming, Princeton Univ. Press, Princeton, NJ, 1957.

2. L. D. Berkovitz, Optimal feedback controls, SIAM J. Control Optim. 27 (1989), 991-1006.

3. V. G. Boltyanskii, Sufficient conditions for optimality and justification of the dynamic programming method, SIAM J. Control Optim. 4 (1966), 326-361.

4. L. Cesari, Optimaization theory and applications, Springer, New York and Berlin, 1983.

5. F. H. Clarke and R. B. Vinter, Local optimality conditions and Lipschitzian solutions to the Hamiltonian-Jacobi equation, SIAM J. Control Optim. 21 (1983), 856-870.

6. W. H. Fleming and R. W. Rishel, Deterministic and stochastic optimal control, Springer, New York and Berlin, 1975.

7. A. Nowakowski, Field theories in the modern calculus of variations, Trans. Amer. Math. Soc. 309 (1988), 725-752. 